\title{
CONSTRUCCIÓN DE UN DISPOSITIVO DE BAJO COSTO COMO APOYO A PERSONAS CON DISCAPACIDAD VISUAL UTILIZANDO REALIDAD AUMENTADA.
}

\section{CONSTRUCTION OF A DEVICE SUPPORT LOW AS VISUALLY IMPAIRED USING AUGMENTED REALITY}

\author{
Samir Castaño, ${ }^{* 1}$, Yeivi J. Peinado ${ }^{2}$, Edilberto Carmona ${ }^{3}$. \\ Recibido para publicación: 16 febrero 2013 - Aceptado para publicación:20 abril 2013
}

\section{RESUMEN}

El presente trabajo de investigación tiene como finalidad la creación de un dispositivo de bajo costo (ARGLASS) como apoyo a personas con discapacidad visual en la identificación de objetos dentro de una vivienda. Para el desarrollo del trabajo se estableció en primer lugar la recolección de información de las principales dificultades en términos de desplazamientos de las personas con discapacidad visual, así como una análisis de las tecnologías a utilizar, en posteriormente, se definieron los requerimientos del sistema, tanto a nivel de hardware como a nivel de software, y en la tercera fase se construyó el dispositivo y su respectiva integración con el software y finalmente se realizaron las pruebas de funcionamiento del sistema en la población objetivo.

Palabras claves: Realidad Aumentada, identificación de objetos, cámara, dispositivo, discapacidad.

\footnotetext{
${ }^{1}$ Especialista en informática y telemática,Magister en software libre, Profesor Auxiliar Universidad de Córdoba, Montería - Colombia samircastano@yahoo.com

2 Ingeniera Sistemas, Universidad de Córdoba, Montería - Colombia yeivimix@hotmail.com

3 Ingeniero Sistemas, Universidad de Córdoba, Montería - Colombia edybrand03@hotmail.com
} 


\section{ABSTRACT}

The present research is aimed at creating a low cost (ARGLASS) device that serves as assistant support for identifying objects within a housing to persons with visual disabilities total and partial that dominate their environment, using a system that uses augmented reality as a tool to deliver information based in audio about these objects, allowing these users recognize their space as if they were seeing.

Keywords: aumented reality, disability, objects identification, camera.

\section{INTRODUCCIÓN}

Según datos estadísticos registrados por el departamento administrativo nacional de estadísticas (DANE) mediante el censo del año 2005 existe en Colombia un total de 1.143.992 personas que presenta dificultades visuales cuyas causas pueden ser variadas, desde una enfermedad crónica, infecciones, golpes, hasta problemas congénitos [4], cifras que van en constante aumento cada día. Sin embargo, las tecnologías existentes que se dirigen a la asistencia de esta población, resultan ser poco ventajosas en comparación con las grandes necesidades que enfrentan, sin incluir el alto costo que genera la utilización de las mismas. Según el Registro de localización y caracterización del Ministerio de Salud y Protección Social en 2012 se muestra que el $87.8 \%$ de la población con discapacidad visual está clasificada en los niveles I y II del SISBEN [2], lo cual demuestra que esta población no dispone de los recursos necesarios para adquisición de dichas herramientas, para mejorar la situación de estas personas, hace falta desarrollar herramientas tecnológicas que les permitan desenvolverse de forma independiente en sus hogares y trabajo, etc.

En la actualidad se han desarrollado proyectos de investigaciones que han dado su aporte a esta población con discapacidad. Entre estos se puede mencionar "Sistema de posición y orientación móvil para personas ciegas en ambientes cerrados" elaborado en la
Universidad de Chile. La aplicación se basó en el uso de tecnología WiFi en conjunto con la representación previa del ambiente [11]. Otra investigación realizada en este mismo país, se centró en la utilización de realidad aumentada con el objetivo de diseñar, desarrollar y evaluar un sistema basado en interfaces de audio para asistir a un usuario ciego en tópicos de movilidad y orientación[12]. De igual manera, en el Instituto de Tecnología de Massachusetts, investigadores del grupo Fluid Interfaces, implementaron la realidad aumentada para la creación de un dispositivo llamado Eyering [9], un anillo con una cámara integrada que grabaría las cosas que se señalan para brindar información auditiva sobre ellas. Su principal objetivo es ayudar a personas invidentes a realizar tareas cotidianas, como ir de compras. Con esto, se evidencia una vez más de que el concepto de realidad aumentada está tomando fuerza en el mundo tecnológico.

Un proyecto de investigación científica utiliza la tecnología RFID para la creación de un sistema de identificación de objetos para personas invidentes mediante la reproducción de una descripción auditiva de los mismos, proporcionando a las personas invidentes la posibilidad de mejorar su calidad de vida [6]. Investigadores de la Universidad Carlos III de Madrid desarrollaron un sistema que, integrado en unas gafas de realidad virtual, ayudan a las personas con discapacidad visual moderada a moverse por su entorno. La aplicación detecta la distancia y forma de los objetos e interactúa 
con el usuario mediante un código de colores. La aplicación fue elaborada en el marco del proyecto de ayudas técnicas integradas para discapacidades visuales. [5]

Finalmente, en Colombia también se han realizado estudios enfocados a ayudar a personas con discapacidad visual, como en el caso de la Universidad Tecnológica de Pereira, que propuso un prototipo experimental de un sistema de detección de objetos por medio de imágenes y sensores para invidentes llamado

"domis" [3]. Esta investigación, hizo uso de teorías y tecnologías ya existentes como los sensores y cámaras para ubicar un objeto en un entorno y brindar información sobre su distancia mediante información sonora y vibratoria.

El objetivo central de este trabajo se orienta en primera medida en la construcción de un dispositivo de bajo costo dada las condiciones de pobreza que existen en la población con discapacidad. Para ello se opta por la utilización de componentes de muy bajo costo como son una cámara web y un par de audífonos que en conjunto con la construcción de una herramienta de software basada en realidad aumentada permita se asequible por esta población.

\section{MATERIALES Y MÉTODOS}

\subsection{Estudio de necesidades en la población}

Como punto central de esta fase, se realizó una recopilación de información para conocer las necesidades y particularidades de las personas con discapacidad visual en términos de desplazamiento y ubicación de objetos dentro de un recinto, así mismo se estableció una encuesta para conocer el grado de conocimiento y uso de las nuevas tecnologías.

\subsection{Requerimientos del sistema}

Dentro de esta fase se especificaron los requerimientos esenciales o primordiales para el funcionamiento del sistema, teniendo como objetivo facilitar la vida a las personas con discapacidad. Para llevar cabo este proceso se desarrollaron dos (2) etapas:
Identificación de los componentes de hardware a utilizar en el proyecto

Definición de requisitos funcionales del software.

\subsection{Construcción del dispositivo y aplicación}

En esta fase se especificaron las directrices pertinentes al proceso de construcción tanto de hardware y software, para ellos se estipularon dos (2) etapas:

Interconexión de los dispositivos físicos, cámara web, audífonos y computador personal.

Diseño y desarrollo de una aplicación basada en realidad aumentada como mecanismo de comunicación entre los dispositivo físicos

\section{RESULTADOS Y DISCUSIÓN}

\subsection{Hardware}

El sistema consta de un dispositivo de bajo costo, que permite identificar objetos relacionados a través de marcadores [10] por medio de una cámara web entregando información auditiva sobre el tipo de objeto reconocido. La cámara fue incorporada a unas gafas convencionales para que ésta pudiese ser utilizada por el usuario a la altura de los ojos y se le adaptaron los audífonos adecuados para la salida de la información.

El dispositivo obtenido (Ver Figura 1) es conectado a un ordenador portátil mediante conexión USB con el fin de brindar una mayor comodidad a la persona con discapacidad.

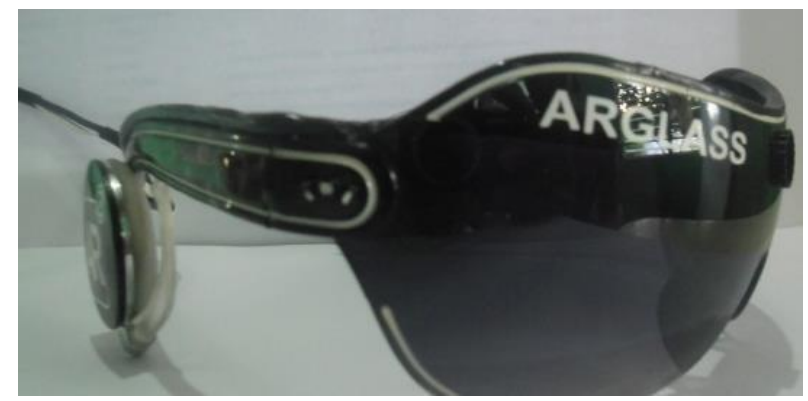

Figura 1. Dispositivo Final 


\section{$3.2 \quad$ Software}

El desarrollo de la aplicación está basado en un modelo de arquitectura de tres capas a razón de establecer independencia entre cada uno de los componentes de la aplicación (Ver Figura 2), representada en los siguientes elementos:

Capa de Presentación (GUI)

Capa Lógica

Capa de Persistencia.

La primera capa llamada presentación, corresponde a la interfaz de usuario construida especialmente para ser visualizada en Flash

Player, usando "widgets" (componentes gráficos para ventanas) de la clase Sprite de ActionScript, que proporciona una variedad de propiedades, métodos y eventos, para graficar componentes de interfaz de usuario (IU).

La segunda capa concierne a la capa lógica de la aplicación, en ella se define cada una de las clases desarrolladas para el manejo de sonidos y la importación de datos a través de archivos de persistencia XML, así como la clase para la captura de eventos generados por cada widgets personalizado, además del uso de librerías que permiten el reconocimiento de marcadores usando Realidad Aumentada.

Finalmente, la capa de persistencia se enfoca en almacenar cada uno de las de rutas de los diferentes archivos de audio que se utilizaran en la aplicación, así como también los parámetros de configuración de la cámara y los respectivos archivos que guardan la codificación de los marcadores.

De igual forma es necesario aclarar la necesidad de programas externos requeridos para la ejecución del software como son la máquina virtual de flash, y el sistema operativo que soporta todos los elementos anteriores.

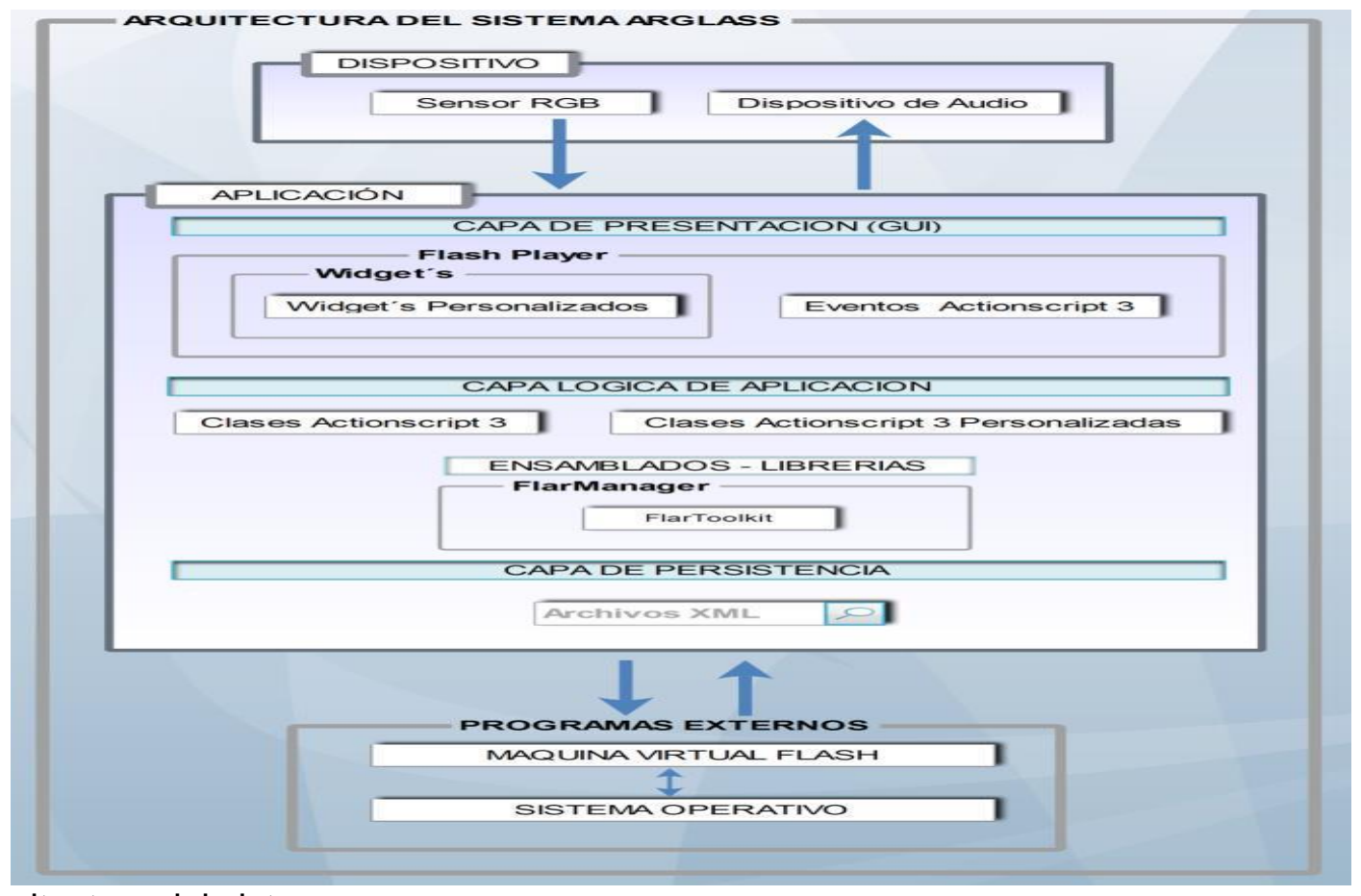

Figura 2. Arquitectura del sistema 
Es de anotar que para el desarrollo de la aplicación se utilizó ActionScript3 [1], un lenguaje de programación orientado a objetos (OOP) que cuenta con elementos para el manejo de documentos XML, expresiones regulares, espacios de nombres, entre otros de igual se utilizaron herramientas tales como: Flex SDK 3.3, para compilar y depurar la aplicación. Gedit, editor de textos que permitió la construcción de las clases necesarias para el desarrollo de la aplicación. FLARToolkit [7], librería utilizada para el reconocimiento y detección de marcadores. FlarManager [8], permitió la gestión de múltiples marcadores necesarios para la identificación de cada objeto

\subsection{Pruebas y Discusión}

El dispositivo fue evaluado principalmente a través de diferentes pruebas que permitieron determinar las distancias óptimas en las que los objetos eran detectados a plenitud. Este procedimiento se realizó de manera continua para verificar que el sistema lograba identificar los objetos a la misma distancia (Ver Figura 3) y a su vez permitió calcular la duración promedio del suministro de energía de la batería del computador portátil, la cual fue aproximadamente de cuatro (4) horas, que determina el tiempo de utilización del sistema. Otra prueba realizada fue determinar si las distancias obtenidas eran afectadas por las dimensiones del marcador y condiciones de luminosidad del ambiente, obteniendo diferentes resultados cuando estos eran modificados 0 cuando se alteraban las condiciones de luminosidad

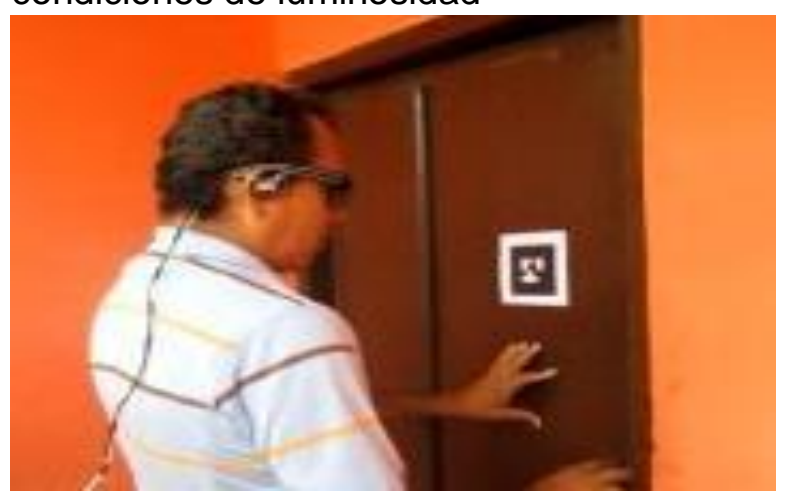

Figura 3. Usuario Utilizando Dispositivo
Es preciso anotar que dentro de entornos conocidos, los usuarios se desenvolvieron con mayor facilidad haciendo uso del dispositivo debido a que tenían conocimiento sobre la distribución espacial de su casa, permitiendo que éste describiera los objetos que el usuario enfocaba. En el caso del entorno desconocido, los usuarios tuvieron dificultades al momento de enfocar dichos objetos, debido a que no conocían el lugar en donde se encontraban. Para comprobar si se obtenían los mismos resultados que en su vivienda, se les realizó un recorrido indicándoles cada uno de los objetos que se encontraban en ella, logrando de esta manera que se adaptarán al espacio. Seguidamente haciendo uso del dispositivo pudieron recorrer el lugar y localizar los objetos casi igual como si estuviesen en su lugar de residencia. Por esta razón, se comprueba que el dispositivo es más eficiente cuando los usuarios lo utilizan en ambientes conocidos.

\section{CONCLUSIÓN}

Se ha presentado un dispositivo de bajo costo que permite identificar objetos dentro de una vivienda a personas con discapacidad visual mediante la utilización de realidad aumentada como herramienta tecnológica.

Con el dispositivo desarrollado, se obtuvieron resultados que fueron de mucha ayuda para este tipo de población, puesto que lograron interactuar de una manera más segura con su entorno al escuchar el tipo de objeto que se ha identificado, previniendo obstáculos que podrían ser representados por los mismos objetos. Además, permitió que el desarrollo de sus actividades diarias fuese más fácil de realizar.

Se espera seguir mejorando con el fin de que el dispositivo pueda cubrir otras necesidades de estas personas, como especificarle la distancia y la posición en la que se encuentran los objetos con respecto a ellos. 
Esta iniciativa no se convirtió en la solución definitiva a todos sus problemas, pero si representó una alternativa que les sirvió como apoyo para el mejoramiento de su calidad de vida. nas-gafas-indican-los-obstaculos-apersonas-con-discapacidad-visual

[6]. El valor de la realidad aumentada (2012),Marker AR. Consultada 10 diciembre 2013. http://www.aumentaty.com/es/conten t/marker-ar

[7]. FlarToolKit (2010), FlarToolKit. Consultada 18 diciembre 2013. http://www.libspark.org/wiki/saqoosh a/ FLARToolKit/en

[8]. FlarManager (2010), FlarManager. Consultada 20 diciembre 2013. http://www.libspark.org/wiki/saqoosh a/ FLARToolKit/en

[9]. Nanayakkara S. C Shilkrot R. and Maes P (2012), EyeRing: A Fingerworn Assitant. International Conference Human Factors in Computing (CHI).

[3]. Cortes J. Castaño L. Morales W , Prototipo experimental de un sistema de detección de objetos por medio de imágenes y sensores para invidentes, ingeniero en meca trónica, Universidad tecnológica de Pereira, Pereira

[4]. Discapacidad Personas con limitaciones permanentes, departamento administrativo nacional de estadística, edición 1, Bogota D.C Septiembre 8 2006, http://www.dane.gov.co/files/censo20 05/discapacidad.pdf

[5]. Discapacidad visual (2012), Unas gafas indican los obstáculos a personas con discapacidad visual. Consultada 10 diciembre 2013. http://www.agenciasinc.es/Noticias/U
[10]. Ortiz L, Sistema de identificación de objetos para personas invidentes usando la tecnología RFID. Ingenius. n. 8 , pp. 38-44. ISSN: 1390-650X.

[11]. Sáenz C Mauricio A, Sistema de posición y orientación móvil para personas ciegas en ambientes cerrados, Magíster en ciencias, universidad de chile, Santiago de chile

[12]. Tadres A, Utilización de realidad aumentada e interfaces basadas en audio para facilitar la movilidad y orientación de personas ciegas, ingeniero civil de computación, Universidad de chile, Santiago de Chile 